\title{
A automedicação e os acadêmicos da área de saúde
}

\author{
Self-medication and health academic staff
}

Daniela Silva de Aquino ${ }^{1}$

JoséAugusto Cabral deBarros ${ }^{2}$

Maria Dolores Paes da Silva ${ }^{3}$

${ }^{1}$ Departamento deFarmácia,

Abstract Asself-medication is a worldwide problem of public health, this article had as objective to evaluate the behavior of the future health professionals with regard to the drugutilization, particularly, to the self-medication practice. 223 academic students from a public university in RecifeCity wereintervi ewed, selected by proportional share in each course. A questionnaire on the drug utilization was applied, 15 days before the interview. From the 223 students, $65.5 \%$ reported drug use in the last 15 days. $42.3 \%$ used drugs through the physician's prescription and $57.7 \%$ through the self-medication. The most used drugs were theanalgesics (24.0\%) and vitamins (18.9\%) and the main reason was pain $(30.5 \%)$. The drug utilization among academic students in Recife City is high but it is inside the parameter according to others analysis. H owever we have expected a low and more rationalized utilization.

Key words Drug utilization, Self-medication, Health academic staff
Faculdades Integradas da

Vitória deSanto Antão. Rua Real da Torre 255A/202,

M adalena. 50610-000

Recife PE.

aquino.daniela@hotmail.com
Resumo Tendo em vista que a automedicação é um problema de saúde pública mundial, esteartigo teve como objetivo avaliar o comportamento dos futuros profissionais de saúde com relação à utilização de medicamentos, particularmente, à prática da automedicação. A amostra foi composta por 223 estudantes da área de saúde de uma universidade pública no município do Recife, selecionados por partilha proporcional a cada curso. Foram feitos questionamentos sobre o uso de medicamentos, nos últimos quinze dias que antecederam à coleta de dados. $65,5 \%$ dos entrevistados confirmaram ter feito uso de medicamentos nos últimosquinze dias, 42,3\% por meio deuma prescrição médica e 57,7\% através da automedicação. Os medicamentos mais utilizados foram os analgési $\cos (24,0 \%)$ eas vitaminas (18,9\%) e o motivo mais apontado foi a dor $(30,5 \%)$. 0 consumo de medicamentos entre os estudantes universitários no município do Recife é elevado, mas encontra-se dentro dos parâmetros observados por outros estudos. Todavia, por se tratar de profissionais da área desaúde, esperava-se que o consumo fosse menor e mais racionalizado.

Palavras-chave Utilização de medicamentos, Automedicação, Acadêmicos de saúde 
Introdução

A automedicação é uma forma importante de cuidados pessoais e evidências mostram que é a forma mais comum de resposta a sintomas ${ }^{1}$. Fatores diversos, como a medicalização e as estratégias promocionais da indústria farmacêutica, podem contribuir para a efetivação de práticas e desejos "irracionais" de utilização de medicamentos por indivíduos ou populações². No Brasil, pelo menos 35\% dos medicamentos são adquiridos por automedicação ${ }^{3}$.

"O consumo é algo inerente ao homem", havendo uma relação entre as transformações da sociedade e o fenômeno do consumo. Sendo assim, o medicamento não está desvinculado dessa característica social. Diferentemente de outras épocas históricas, o capitalismo pós-moderno incentiva o consumo através da publicidade e da ideia da substituição do "prazer vicário do ter sobre o ser"4.

$\mathrm{Na}$ sociedade moderna, à medida que 0 acesso ao consumo foi convertido no objetivo principal para o desfrute de níveis satisfatórios de bem-estar, bons níveis de saúde passaram a ser vistos como possíveis na estreita dependência do acesso a tecnologias diagnóstico-terapêuticas. A eficácia e a efetividade das mesmas se tornaram cada vez mais subordinadas ao grau de esmero técnico alcançado $0^{3,5}$.

Este fenômeno caracteriza a medicalização e uma das grandes consequências do seu incremento éa intensificação da dependência. As pessoas pretendem resolver seus problemas, sejam eles médicos ou não, recorrendo aos serviços oferecidos pelo sistema de saúde?.

Por outro lado, na tentativa de definir o consumo em favor de seus próprios produtos, as indústrias lançam mão de diversas práticas promocionais que visam atingir o médico, o farmacêutico, o balconista, o dono da farmácia, os pacientes e os consumidores em geral ${ }^{3,6,7}$.

A propaganda massiva e a facilidade de acesso a medicamentos em farmácias e supermercados dão a impressão de que são produtos livres de riscos. Além disso, estimulam o uso indiscriminado, o que nem sempre resulta nos efeitos prometidos, e expõe os consumidores a reações indesejadas, às reações adversas, semprecrescentes devido ao consumo elevado de medicamentos que se observa na atualidade. A crença deque a pílula seja capaz de eliminar ou, pelo menos, amenizar estes problemas é fator complementar e primordial para o consumo ${ }^{8}$.
Os médicos são o principal alvo das técnicas promocionais da indústria farmacêutica. Afinal, a escolha deum ou outro medicamento depende fundamentalmente deles e sua influência atinge até mesmo o consumo por automedicação. Os argumentos mais utilizados são, em geral, eficácia, segurança, comodidade, rapidez de ação e alta tolerabilidade ${ }^{8}$.

Embora a automedicação seja uma necessidade, tendo inclusive uma função complementar aos sistemas de saúde, particularmente em países pobres, é evidente que este hábito, utilizado de maneira inadequada, pode ter como consequência efeitos indesejáveis, enfermidades iatrogênicas emascaramento de doenças evolutivas, representando, portanto, problema a ser prevenido 9 .

0 ideal, portanto, é utilizar o medicamento apenas quando imprescindível e recomendado por um profissional especializado.

Sendo assim, este estudo tevepor objetivo verificar o comportamento dos futuros profissionais de saúde com relação à utilização de medicamentos, particularmente, à prática da automedicação, uma vez que deverão ser os responsáveis pela orientação e educação de pacientes e população quanto ao uso correto de medicamentos.

\section{Métodos}

Foi realizado um estudo do tipo transversal composto por uma amostra de 223 estudantes dos cursos da área de saúde de uma universidade pública do município do Recife. Utilizou-seuma amostra casual simples com partilha proporcional. Para cálculo do tamanho da amostra, considerou-se o fato de não se conhecer o percentual da população que possuia a característica estudada. Assim, conforme recomendação de Arkin e Colton ${ }^{10}$, utilizou-se o valor de $50 \%$. Este procedimento maximizou o tamanho da amostra, diminuindo, portanto, o erro amostral.

Desta forma, considerou-se o número total dealunos que estavam matriculados em disciplinas no Centro de Ciências da Saúde, excluindose aqueles com matrículas vínculo e os que tinham efetuado trancamentos. Posteriormente, procedeu-se à proporcionalidade, calculando 0 percentual que cada curso correspondia no universo do centro acadêmico, e aplicou-se, então, este percentual ao valor amostral, obtendo-se a seguinte distribuição: educação física (39); enfermagem (21); farmácia (27); fisioterapia (18); fonoaudiologia (5); medicina (56); nutrição (16); 
odontologia (30); terapia ocupacional (11), totalizando 223 estudantes.

As informações foram coletadas por meio de um questionário estruturado, previamente validado entrealunos da mesma instituição. Os participantes foram abordados no seu centro acadêmico aleatoriamente e as entrevistas tiveram continuidade até serem completados o número dequestionários necessários a cada curso. A aplicação dos questionários se deu no período de maio a junho de 2005, com consentimento pré vio dos estudantes. Foram feitos questionamentos sobre o uso de medicamentos nos últimos quinze dias que antecederam à coleta de dados.

Considerou-se como automedicação todos aqueles medicamentos autoindicados, indicados por parentes, amigos, balconistas ou outras pessoas não formalmente habilitadas para prescrever.

Os dados coletados foram duplamente digitados no programa Epilnfo, versão 6.04, para identificação e correção dos erros de digitação. Os medicamentos foram classificados de acordo com o código anátomo-terapêutico-químico $(\text { ATC })^{11}$.

O projeto de pesquisa foi aprovado pelo ComitêdeÉtica em Pesquisa do Centro de Ciências da Saúde da Universidade Federal de Pernambuco de acordo com a Resolução n 196/96 do Conselho Nacional de Saúde.

\section{Resultados}

O perfil dos entrevistados evidenciou que o maior número departicipantes era do sexo feminino $(61,4 \%)$. Com relação ao grupo etário, havia maior número de participantes entre 20 e 22 anos (57,0\%). 39,0\% cursavam entre o quarto eo sexto período acadêmico. 0 estado civil predominante, em mais de $98,0 \%$ dos casos, era o solteiro. Pouco mais de $42,0 \%$ dos participantes apresentaram uma renda familiar entre seis e dez salários mínimos (Tabela 1).

$65,5 \%$ dos entrevistados confirmaram ter feito uso de medicamentos nos últimos quinze dias (Figura 1). Com relação à fonte que indicou o(s) medicamento(s), a percentagem dos que referiram a existência de uma prescrição médica foi de $42,3 \%$ e aqueles que se automedicaram, ainda que influenciados pela mídia, por parentes, amigos, balconistas de farmácia, dentre outros, somaram $57,7 \%$ das pessoas (Figura 2 ).

Os medicamentos mais utilizados nos últimos quinze dias foram os anal gésicos e as vitaminas. 0 grupo terapêutico "analgésicos/antitér- micos" foi citado por $24,0 \%$ das pessoas e "vitaminas/antianêmicos", por 18,9\%. Como algumas pessoas fizeram uso de mais de um medicamento, o n, neste caso, foi de 233 (Tabela 2).

Dentre os motivos que levaram os estudantes a fazerem uso de medicamentos nos últimos quinze dias, destacou-se a dor (30,5\%). Imedi-

Tabela I. Perfil epidemiológico e sociodemográfico da amostra.

\begin{tabular}{lrr}
\hline \multirow{2}{*}{ Características } & \multicolumn{2}{c}{ Acadêmicos } \\
\cline { 2 - 3 } sociodemográficas & $\mathrm{n}$ & $\%$ \\
\hline Sexo & 86 & 38.6 \\
M asculino & 137 & 61.4 \\
Feminino & & \\
Grupo etário & 39 & 17.5 \\
17-19 anos & 127 & 57.0 \\
20-22 anos & 57 & 25.5 \\
23 e + anos & & \\
Período no curso & 82 & 36.8 \\
1-3 semestre & 87 & 39.0 \\
4-6 semestre & 54 & 24.2 \\
7 e + semestre & & \\
Estado civil & 219 & 98.2 \\
Solteiro & 4 & 1.8 \\
Casado & & \\
Renda familiar (SM & \\
Até 1 SM & 3 & 1.3 \\
2 a 5 SM & 41 & 18.4 \\
6 a 10 SM & 94 & 42.1 \\
>10 SM & 83 & 37.2 \\
\hline
\end{tabular}

${ }^{*} \mathrm{SM}=$ salário mínimo $=\mathrm{R} \$ 300,00$.

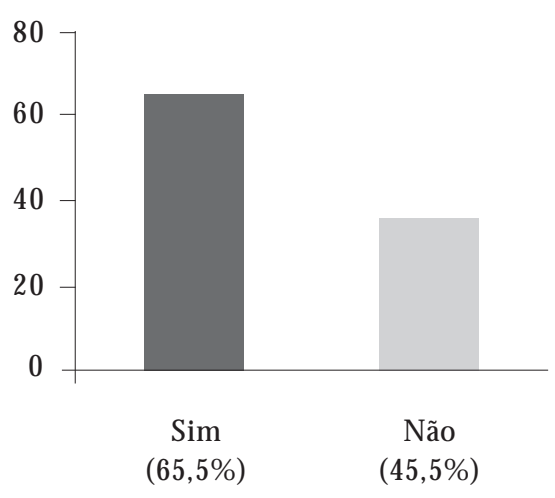

Figura 1. Frequência do uso de medicamentos nos últimos quinze dias. 
atamente depois, surgiram a prevenção de resfriado/suplementação alimentar (12,7\%) e resfriados (10,8\%) (Tabela 3). Ademais, a maioria $(70,8 \%)$ justificou o uso de medicamentos sem receita médica pelo conhecimento acerca do medicamento (uso há muito tempo, prescrição mé dica anterior e uso frequente por toda a família). $18,6 \%$ dos partici pantes alegaram falta de tempo de ir a um médico eum número menor $(10,6 \%)$

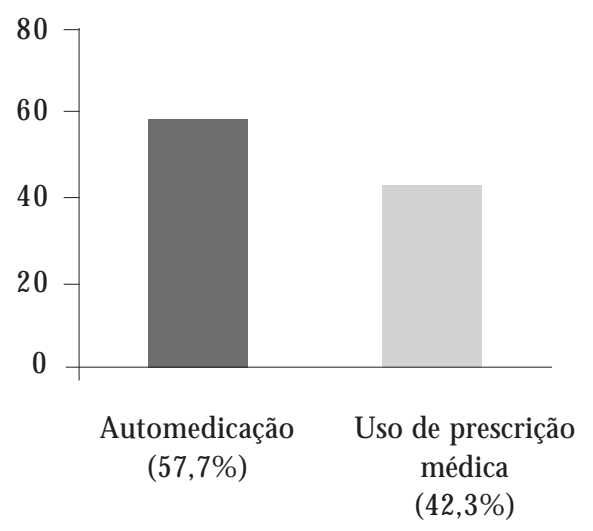

Figura 2. Fonte de indicação dos medicamentos.

Tabela 2. Medicamentos mais utilizados pelos entrevistados nos últimos quinze dias.

\begin{tabular}{lrr}
\hline \multirow{2}{*}{ Medicamento* $^{*}$} & \multicolumn{2}{c}{ Acadêmicos } \\
\cline { 2 - 3 } & \multicolumn{1}{c}{$\mathrm{n}$} & \multicolumn{1}{c}{$\%$} \\
\hline Vitamina/antianêmico & 44 & 18.9 \\
Analgésico/antitérmico & 56 & 24.0 \\
AlNE/antirreumático & 18 & 7.7 \\
Antibiótico/antimicótico/antiviral/ & 16 & 6.9 \\
antiparasitário de uso sistêmico & & \\
Preparações para tosse e resfriado & 11 & 4.7 \\
Antibiótico/antimicótico/corticóide & 10 & 4.3 \\
de uso tópico & & \\
Antiasmático/descongestionante & 8 & 3.4 \\
Antidepressivo/ansiolítico/ & 9 & 3.9 \\
anticonvulsivante & & \\
Antiespasmódico & 7 & 3.0 \\
Outros & & \\
Total & 54 & 23.2 \\
\hline
\end{tabular}

* Foi utilizada a classificação ATC, proposta pela OM S.

*Agrupou hormônio sexual, outroshormônios, miorrelaxante, expectorante, anti-histamínico/ corticóide de uso sistêmico, antiácido/antiúlcera, anorexígeno, betabloqueador, antiglicêmico. apontou o difícil acesso ao sistema de saúde, razões financeiras, comodidade e a não necessidade de buscar cuidados médicos.

\section{Discussão}

O medicamento enquanto símbolo de saúde, até mesmo na medida em que o usuário leigo não tem idéia de como ele funciona no organismo, é a possibilidade mágica com a qual a ciência, por intermédio da tecnologia, tornou acessível este desejo de consumi-lo, através de uma pílula ou algumas gotas, sob a forma de prevenção, remissão e triunfo definitivo (na cura), reproduzindo no dia a dia (no controle) sobre o cortejo de males do corpo e da alma que afetam o homem e sobre as "carências" ou limitações inerentes à condição humana: medicamentos geriátricos contra a perda da memória, vitaminas contra a calvície, etc. ${ }^{12}$.

Verificando-se a utilização de medicamentos nos últimos quinze dias que antecederam à entrevista, $65,5 \%$ dos entrevistados confirmaram 0 uso. Resultados semelhantes foram encontrados em estudos realizados no sul do Brasil ${ }^{13,14}$. Parece que as pessoas precisam consumir para sentir-sebem, preencher seus vazios, eo medicamento aparece aqui como outros bens de consumo, como se não apresentasse riscos e pudesse ser consumido livremente.

N ossos achados apontam que, entre aqueles que consumiram medicamentos nos últimos quinze dias, $42,3 \%$ o fizeram através da prescrição médica e 57,7\% através da automedicação,

Tabela 3. Motivos mais citados pelos entrevistados para o uso de medicamentos nos últimos quinze dias.

\begin{tabular}{lrr}
\hline \multirow{2}{*}{\multicolumn{1}{c}{ Sintoma }} & \multicolumn{2}{c}{ Saúde } \\
\cline { 2 - 3 } & \multicolumn{1}{c}{$\mathrm{n}$} & \multicolumn{1}{c}{$\%$} \\
\hline Dor & 65 & 30.5 \\
Prevenção/suplementação & 27 & 12.7 \\
Resfriado & 23 & 10.8 \\
Problemas com a garganta & 9 & 4.2 \\
Febre & 10 & 4.7 \\
Outros & 79 & 37.1 \\
Total & 213 & 100.0 \\
\hline
\end{tabular}

*Agrupou anticoncepção, acne, tosse, alergia, estresse, gastrite, diarréia e problemas musculares. 
sendo maior no grupo feminino $(66,5 \%)$. Isto pode ser explicado em parte pela maior exposição das mulheres à medicalização em todas as fases de sua vida, maior procura por cuidados médicos e campanhas educativas mais direcionadas a elas. Simões et al..$^{15}$, avaliando as características do consumo de medicamentos na população urbana deAraraquara (SP), verificaram que $42,1 \%$ dos medicamentos utilizados foram adquiridos sem prescrição médica.

Os analgésicos lideraram a preferência dos entrevistados (24,0\%). Arrais et al. ${ }^{9}$ e Bertoldi et al. ${ }^{14}$ também apresentaram os anal gésicos como os medicamentos mais utilizados em seus estudos com resultados próximos ao nosso, 21,6\% e $26,6 \%$, respectivamente. A segunda classe terapêutica mais utilizada pela população em estudo foram as vitaminas $(18,9 \%)$. O utros estudos apresentaram resultados bastanteinferiores, com percentagens variando de $5,0 \%$ a 7,7\% $\%, 13-15$. Alguns fatores como o fácil acesso aos medicamentos e a propaganda em massa, especialmente das vitaminas, que tendem a associá-las à beleza, jovialidade, vigor físico, aliados à curiosidade, comodidade e busca pelo alívio imediato dos sintomas, contribuem bastante para a prática da automedicação.

Com relação aos motivos que levaram as pessoas a utilizarem medicamentos nos últimos quinze dias que precederam a coleta de dados, a nossa pesquisa apontou a dor como o motivo mais citado (30,5\%), especialmente cefaléia, dores musculares e dismenorréia, seguido da prevenção de resfriados/suplementação alimentar, $(12,7 \%)$ e resfriados (10,8\%). No estudo de $\mathrm{Ar}$ rais et al. ${ }^{9}$, todas as expressões clínicas de dor somaram $24,2 \%$ dos motivos que geraram a automedicação.

0 uso rotineiro de analgésicos e vitaminas constitui uma prática que parece dever-seà ideia deque se tratam de produtos inofensivos. Inclusive com relação às vitaminas, muitos estudantes não as viam como medicamentos, pois negavam o uso das mesmas, e, no entanto, relatavam fazer uso de vitaminas duas ou mais vezes por semana ou mesmo diariamente. Portanto, fica o alerta, o mesmo medicamento que alivia os sintomas, traz al gum conforto ecura, também mata.

Verifica-setambém o uso deantibióticos, que embora devessem ser dispensados com apresentação de receita médica, são ven didos livremente. O seu uso deforma inadequada tem contribuído maciçamente para o mecanismo de resistência bacteriana em todo o mundo, sendo, portanto, um problema de saúde pública mundial.
É ainda preocupante o uso de substâncias de venda controlada como os anorexígenos, antidepressivos e ansiolíticos. No caso dos anorexígenos, não se conhece exatamente seu mecanismo de ação como supressores de apetite. Julgase que o efeito principal destes fármacos é sobre o centro de controle do apetite no hipotálamo; isso resulta na redução da fome por alteração do controle químico da transmissão do impulso nervoso. Devem ser utilizados apenas como adjuvantes no tratamento da obesidade ${ }^{16}$. Mas, na prática, não éisso que vem ocorrendo, pois esses produtos têm sido empregados como tratamento primário para promover a perda de peso, na busca do corpo perfeito.

Com relação ao uso de ansiolíticos e antidepressivos, constatou-se ser o estresse o motivo apontado pela maioria dos estudantes para uso dos mesmos. Ora, tem-se aqui um típico problema da sociedade moderna, em que a "solução" foi buscada na "pílula". Não seria mais adequado diminuir o ritmo das atividades, dedicar mais tempo ao lazer e ao descanso? Há quese considerar que não é uma tarefa fácil; no entanto, ainda que lentamente, as pessoas devem tentar promover as mudanças, não recorrendo, de pronto, à solução mais rápida: "o medicamento".

\section{Conclusões}

O consumo de medicamentos entre os estudantes universitários no município do Recifeéelevado, mas encontra-se dentro dos parâmetros observados por outros estudos. Todavia, por se tratar de futuros profissionais da área de saúde, esperava-se que o consumo fosse menor e mais racionalizado. $M$ as, parece queéjustamente esse maior conhecimento que os predispõe ao uso de forma inadequada.

Fica difícil, portanto, esperar que estes universitários, especialmente os estudantes de medicina e farmácia, orientem seus pacientes quanto ao uso correto dos medicamentos, se eles próprios parecem não ter consciência dos riscos. Ou seja, desta forma, não representam agentes multiplicadores de cunho educativo, quanto ao uso racional de medicamentos, mas sim, parece que estamos diante de futuros profissionais coniventes com a prática da automedicação e do uso incorreto dos medicamentos. Porém, os estudantes não são detodo culpados; além da herança cultural que trazem consigo, as instituições de ensino pouco têm feito nas suas formações para que eles possam assumir o papel de acolhedores e orien- 
tadores de seus pacientes. Para reverter este quadro, faz-se necessário a incorporação de práticas educativas quanto ao uso correto dos medicamentos, riscos, benefícios, superdosagem, intoxicações, reações adversas, gastos para o sistema de saúde decorrentes de internações devido a problemas relacionados a medicamentos. Só assim, espera-se que a população possa receber cuidados de saúde de qualidade de profissionais competentes e preocupados em preservar a sua saúde.

\section{Colaboradores}

DS Aquino trabalhou na concepção do projeto de estudo, coleta, organização do banco de dados, análise e interpretação dos resultados e redação do artigo. JAC Barros atuou na revisão crítica ediscussão dos resultados. M DP Silva subsidiou na metodologia, análise e interpretação dos resultados.

\section{Referências}

1. Levin LS, Beske F, Fry Jr. Self-medication in Europe. Report on a study of the role of non-prescription medicines. Copenhagen: World Health Organization Regional Office for Europe; 1988.

2. Fabricant SJ, Hirchhorn N. Deranged distribution, perverse prescription, unprotected use: the irrationality of pharmaceuticals in the developing world. Health Policy and Planning 1987; 2(3):204-213.

3. Barros JAC. Propaganda de medicamentos: atentado à saúde? São Paulo: Hucitec/Sobravime; 1995.

4. Rozenfeld S, Porto MA. Vigilância Sanitária: uma abordagem ecológica da tecnologia em saúde. In: Buss PM, Sabroza P, Leal MC, organizadores. Saúde, ambiente e desenvolvimento. São Paulo: Hucitec; Rio de Janeiro: Abrasco; 1992. p. 171-196.

5. Barros JAC. Políticas farmacêuticas: a serviço dos interesses da saúde? Brasília: UNESCO/ANVISA; 2004.

6. Heineck I, Gallina SM, Silva T, Pizzol FD, Schenke EP. Análise da publicidade de medicamentos veiculada em emissoras de rádio do Rio Grande do Sul, Brasil. Cad Saude Publica 1998; 14(1):193-198.

7. Pizzol F, Silva T, Schenkel EP. Análise da adequação das propagandas de medicamentos dirigidas à categoria médica distribuídas no Sul do Brasil. Cad Saude Publica 1998; 14(1):85-90.

8. Nascimento MC. M edicamentos: ameaça ou apoio à saúde? Rio de Janeiro: Vieira e Lent; 2003.

9. Arrais PSD, Coelho HLL, Batista M CDS, Carvalho ML, Righi RE, Arnau JM. Perfil da Automedicação no Brasil. Rev Saude Publica 1997; 31(1):71-77.
10. Arkin H, Colton RR. Tables for statistics. New York: Barnes e Nobles; 1950.

11. World Health Organization. The Selection and U se of Essential M edicines. Technical Report Series no 920, Annex 2: The Anatomical Therapeutic Chemical (ATC) Classification System. Genebra: WHO; 2004.

12. Lefèvre F. $O$ medicamento como mercadoria simbólica. São Paulo: Cortez; 1991

13. Vilarino JF, Soares IC, Silveira CM, Rodel APP, Bortoli R, Lemos RR. Perfil da Automedicação em M unicípio do Sul do Brasil. Rev Saude Publica 1998; 32(1):43-49.

14. Bertoldi AD, Barros AJD, Hallal PC, Lima RC. Utilização de medicamentos em adultos: prevalência e determinantes individuais. Rev Saude Publica 2004; 38(2):228-238.

15. Simões MJ, Farache Filho A. Consumo de medicamentos em região do Estado de São Paulo (Brasil), 1985. Rev Saude Publica 1988; 22(6):494-499.

16. Korolkovas A. Dicionário terapêutico Guanabara. Rio de Janeiro: Guanabara Koogan; 2000.

Artigo apresentado em 18/06/2007 Aprovado em 07/05/2008 